\title{
Rhinoscleroma - A Clinical Mimicker of Malignancy: A Case Report
}

\author{
Santosh $\mathbf{T}^{1 *}$, Manoj Kumar $\mathbf{P}^{2}$, Atanu Kumar $\mathbf{B}^{2}$ and Jayanti $\mathbf{N}^{2}$ \\ ${ }^{1}$ Department of Pathology and Lab medicine, AlIMS, Raipur, Chhattisgarh, India \\ ${ }^{2}$ Department of Pathology, MKCG Medical College, Berhampur, Odisha, India
}

\begin{abstract}
Rhinoscleroma (Scrofulous lupus, scleroma) is an infectious disease caused by a Gram negative bacillus Klebsiella rhinoscleromatis. This is a chronic granulomatous disease which commonly involves the nose and upper respiratory tract, but can involve the oral cavity and soft tissues of the lips. The present case was reported as the presentation was with a mass lesion in the right upper lip and philtrum causing nasal obstruction in a 30 year old man. The lesion clinically simulated a malignant lesion. The histopathological examination revealed granulation tissue with plasma cells and Mikulicz's cells.
\end{abstract}

Keywords: Klebsiella rhinoscleromatis; Mikulicz's cells; Rhinoscleroma; Russell bodies

\section{Abbreviations}

AFB: Acid Fast Bacilli; ENT: Ear Nose Throat Department; ESR: Erythrocyte Sedimentation Rate; PAS: Periodic Acid Schiff's stain

\section{Introduction}

Klebsiella rhinoscleromatis, the causative agent of rhinoscleroma, is a Gram-negative, facultatively intracellular, non-motile, encapsulated bacillus. The organism has a very low infectivity necessitating a chronic exposure for infection. Central America, Africa and the Middle East are endemic, but sporadic cases can also been encountered attributed to immigration [1]. Prevalence is also high in developing countries like India. The factors responsible for this are attributed to poor hygiene, poor access to medications and overcrowded living conditions [2]. Though entire respiratory mucosa can be affected, the organism has an affinity for nasal mucosa and thus nasal cavity is the most prevalent site accounts for $95-100 \%$ of cases [3]. In decreasing order of involvement nasopharynx (18-43\%), larynx (15-40\%), trachea (12\%) and bronchi $(2-7 \%)$ are the other sites [4]. Involvement of oral cavity and soft tissues of upper lip and philtrum are uncommon.

The present case had a mass lesion that involved upper lip, nasal cavity and palatal areas causing airway obstruction, raising clinical suspicion of malignancy.

\section{Case Report}

A 30 year old Hindu male patient came to the ENT outpatient department of MKCG Medical College and Hospital with chief complaint of mass lesion involving the upper lip, philtrum and bilateral nostrils leading to progressive nasal obstruction and impaired sense of smell for past 2 years (Figure 1). He observed an ulcerated nodular lesion in his palate causing difficulty in eating and swallowing since last one year. To start with he noticed a small mass in the right nostril which gradually progressed to fill not only bilateral nostrils but also extended to involve the vestibule crossing the midline over the collumela and adjacent part of the upper lip. The lesion was painful on touch with occasional episodes of unprovoked bleeding. He is a day labourer by profession, drank socially, but never smoked.

General examination findings were unremarkable except mild pallor. There was no lymphadenopathy. He denied of any weight loss or night sweats. On local examination, a pinkish nasal mass filling both the nostrils extending beyond the midline over the collumela,

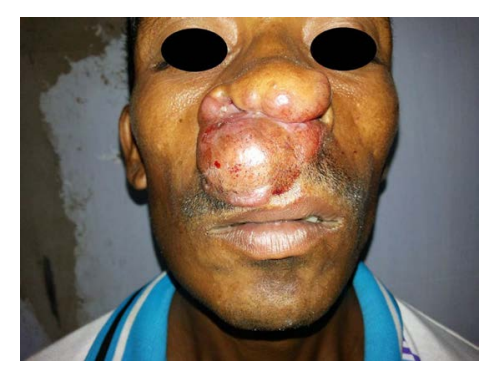

Figure 1: A reddish-grey nasal mass filling both the nostrils, extending beyond the midline over the collumela and adjacent part of the philtrum.

adjacent part of the philtrum and disfiguring the shape of nose. It was tender did not bleed on touch and was firm to hard in consistency. The nasal lesions could not be probed nor evaluated endoscopically due to extreme tenderness. Multiple painless, indurated pinkish erythematous ulcers, covered with slough were observed in gingiva, hard palate and extending upto soft palate (Figure 2).

Routine haematological and biochemical investigation findings were within normal range except mild anemia (Hb-10.5 gm/dl). ESR was $20 \mathrm{~mm}$ in first hour. $\mathrm{Zn}$ stains for tubercular bacilli were negative from the nasal swabs and palatal ulcers. Culture sensitivity of the pus showed growth of Staphylococcus aureus. Radiological evaluation of chest and abdomen revealed no abnormality. From the above findings a possible diagnosis of malignant mass lesion was suspected clinically.

A incisional biopsy for histopathological evaluation revealed squamous epithelial lining with a subepithelial tissue showing a dense, mixed inflammatory cell infiltrate containing plenty of plasma cells with many showing Russell bodies- eosinophilic structures within the cytoplasm of plasma cells and Mikulicz cells (large histiocytes with

*Corresponding author: Santosh T, Department of Pathology and Lab medicine, AlIMS, Raipur, Chhattisgarh, India, Tel: 08895495670; E-mail: born_vss@yahoo.co.in

Received July 11, 2015; Accepted August 17, 2015; Published August 19, 2015.

Citation: Santosh T, Manoj Kumar P, Atanu Kumar B, Jayanti N (2015) Rhinoscleroma - A Clinical Mimicker of Malignancy: A Case Report. J Cytol Histol 6: 365. doi:10.4172/2157-7099.1000365

Copyright: @ 2015 Santosh T, et al. This is an open-access article distributed under the terms of the Creative Commons Attribution License, which permits unrestricted use, distribution, and reproduction in any medium, provided the original author and source are credited. 
Citation: Santosh T, Manoj Kumar P, Atanu Kumar B, Jayanti N (2015) Rhinoscleroma - A Clinical Mimicker of Malignancy: A Case Report. J Cytol Histol 6: 365. doi:10.4172/2157-7099.1000365

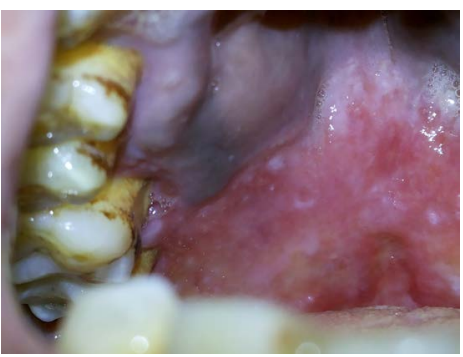

Figure 2: Oral cavity showing pinkish, erythematous ulcers involving the gingiva, hard and soft palate.

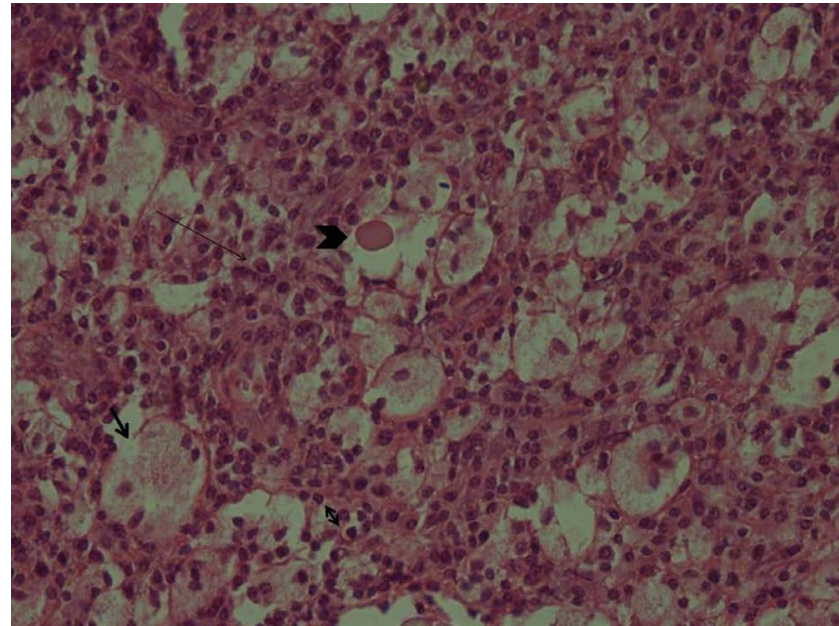

Figure 3: Microscopy showing chronic inflammatory cells i.e. plasma cells, lymphocytes and mikulicz cells i.e. large macrophages with clear cytoplasm containing rod like structures (H\&E, 40X).



Figure 4: Mikulicz's cells contain PAS positive inclusion bodies, corresponds to Klebsiella rhinoscleromatis (PAS, 40X).

numerous vacuoles containing viable or nonviable bacteria) (Figure 3). The Mikulicz cells were PAS positive (Figure 4) and WarthinStarry stain demonstrated that the PAS positive material were rodshaped bacilli within the vacuolated macrophages. The organisms were characterized as Klebsiella based on morphology. The patient was then treated with ciprofloxacin $500 \mathrm{mg}$ twice daily for 12 weeks. On follow up, his airway symptoms gradually improved over last 3 months of treatment with complete remission of mass lesion. Given the significant clinical improvement, the patient chose not to undergo further surgical treatment.

\section{Discussion}

Rhinoscleroma (Scrofulous lupus, scleroma) is a chronic, progressive, inflammatory disease of the upper respiratory tract affecting mainly nasal passages [5]. The term rhinoscleroma was first used in 1870 by Von Hebra and Kaposi while describing a lesion in the nose which they labelled as sarcoma. The Polish surgeon Johann von Mikulicz in 1877 described the histological features of this disease in detail and established its non-neoplastic inflammatory nature. Von Frisch identified the causative agent of this lesion in 1882 as a Gramnegative coccobacillus, now known as Klebsiella rhinoscleromatis [6]. In 1932, Belinov proposed use of term scleroma respiratorium because the pathological process in rhinosclerosis may involve not only upper but also lower airways. In 1961, Steffen and Smith demonstrated that $K$ rhinoscleromatis conformed to Koch's postulates and that it was an etiologic factor in the inflammatory changes typical of scleroma [6].

Rhinoscleroma is more frequent in second or third decades of life with a definite female preponderance. People living in crowded rural areas with poor hygiene and nutritional conditions such as iron deficiency anemia are more prone [7]. The tropical Africa, India, Southeast Asia, Central and South America and Central Europe are endemic regions [8]. Mere infection by $K$. rhinoscleromatis is not enough for the development of the disease as established from the fact that, close contact of the patient with an healthy individuals for many years may not necessarily bring about infection in the latter. This has led to the suggestion that susceptibility of the host is important in development of the disease. Impaired cellular immunity has been detected in these patients but humoral immunity was found to be normal [7]. Rhinoscleroma usually affects the nasal cavity but lesions in larynx, nasopharynx, oral cavity, paranasal sinuses, soft tissues of lips, nose, trachea and bronchi are also seen. $95 \%$ of scleromas are located in the nasal fossae involving the mucosa. Extension to the adjacent skin has also been reported [5,8]. The most common complaint is nasal obstruction followed by rhinorrhea, epistaxis, dysphagia, stridor and dysphonia. The major deleterious effect of rhinoscleroma is the airway obstruction which requires endoscopic treatment [7].

The disease has three distinct clinical and pathological stages namely, catarrhal (inflammatory), proliferative (granulomatous), and fibrotic (sclerotic). Catarrhal stage has no diagnostic histopathological findings so in cases with strong clinical suspicion isolation and identification of the organism from the nasal swab on Mac Conkey agar established the diagnosis [9]. The pathognomonic finding in the Proliferative (granulomatous) stage is the demonstration of Mikulicz cells and plasma cells with Russell bodies in histological sections and positive Warthin-Starry stain. So differentials that are to be kept in mind include bacterial causes such as tuberculosis, actinomycosis and leprosy which can produce granulomas in the upper airways. Fungal infections including histoplasmosis, blastomycosis and sporotrichosis should also be considered. Other differentials consist of mucocutaneous leishmaniasis, malignancy such as basal cell carcinomas, lymphomas, inflammatory lesions such as sarcoidosis and Wegener's granulomatosis [10].

Clinical remission and relapse is a common finding in rhinoscleroma. Treatment option depends on the stage of presentation. With recurrence rate being high, a long duration of antibiotic treatment is advisable. Recommended antibiotics are streptomycin, doxycycline, tetracycline 
Citation: Santosh T, Manoj Kumar P, Atanu Kumar B, Jayanti N (2015) Rhinoscleroma - A Clinical Mimicker of Malignancy: A Case Report. J Cytol Histol 6: 365. doi:10.4172/2157-7099.1000365

and ciprofloxacin. Ciprofloxacin, a fluoroquinolones is an antibiotic with excellent tissue penetration and a broad antibacterial spectrum of action. Its usual dosage is $250-500 \mathrm{mg}$, twice daily for six weeks to six months shows excellent clinical efficacy. It is not recommended in patients under 12 years of age because of the risk of arthropathy [11]. Rhinoscleroma is usually not fatal when diagnosed early and treated promptly. However patients may die from respiratory obstruction or intracranial extension [12].

\section{Conclusion}

Rhinoscleroma is a chronic and indolent Klebsiella infection. It is a long-standing disease that begins in the nasal mucosa, extends to other respiratory tract organs producing airway obstruction and sometimes threatens the patient's life. A diagnosis of rhinoscleroma is made based on histopathological findings. Although it mimics malignancy, rhinoscleroma often responds to treatment with fluoroquinolones, if diagnosis is made early and treated. Hence, communication between the clinician and the pathologist as to the possibility of non-oncological processes can aid in determining the diagnosis and early treatment.

\section{Consent}

A written informed consent has been obtained from the patient for publication of this case report and for any accompanying images. The original copy of the same is available with the corresponding author which can be submitted to the editor-in-chief of this journal on demand.

\section{Competing Interests}

The authors declare that they have no competing interests. There was no financial or non-financial competing interest with any party, person or institute.

\section{Author's Contribution}

TS carried out concepts and design, literature search, participated in clinical study, data acquisition, data analysis and manuscript preparation will stand as guarantor also. MP carried out concepts and design, literature search, manuscript review. $\mathrm{AB}$ participated in clinical study, data acquisition and manuscript review. JN carried out literature search, clinical study and data acquisition.

\section{Acknowledgement}

The authors would like to thank Prof. Debi Prasad Mishra for his constant intellectual and moral support, improvising nature.

\section{References}

1. Miller RH, Shulman JB, Canalis RF, Ward PH (1979) Klebsiella rhinoscleromatis: a clinical and pathogenic enigma. Otolaryngol Head Neck Surg 87: 212-221.

2. Stiernberg CM, Clark WD (1983) Rhinoscleroma-a diagnostic challenge Laryngoscope 93: 866-870.

3. Yigla M, Ben-Izhak O, Oren I, Hashman N, Lejbkowicz F, et al. (2000) Laryngotracheobronchial involvement in a patient with nonendemic rhinoscleroma. Chest 117: 1795-1798.

4. Gaafar HA, Gaafar AH, Nour YA (2011) Rhinoscleroma: an updated experience through the last 10 years. Acta Otolaryngol 131: 440-446.

5. Zhong Q, Guo W, Chen X, Ni X, Fang J, et al. (2011) Rhinoscleroma: a retrospective study of pathologic and clinical features. Otolaryngol Head Neck Surg 40: 167-174.

6. Abalkhail A, Satti MB, Uthman MA, Al Hilli F, Darwish A, et al. (2007) Rhinoscleroma: a clinico-pathological study from the Gulf region. Singapore Med J 48: 148-151.

7. Champs CD, Vellin JF, Diancourt L, Brisse S, Kemeny JL, et al. (2005) Laryngeal Scleroma associated with Klebsiella pneumoniae sub sp.ozaenae. J Clin Microbiol 43: 5811-5813.

8. Shoeib MA (2010) Septal rhinoscleroma. Indian J Plast Surg 43: 219-221.

9. Botelho-Nevers E, Gouriet F, Lepidi H, Couvret A, Amphoux B, et al. (2007) Chronic nasal infection caused by Klebsiella rhinoscleromatis or Klebsiella ozaenae: two forgotten infectious diseases. Int J Infect Dis 11: 423-429.

10. Tan SL, Neoh CY, Tan HH (2012) Rhinoscleroma: a case series. Singapore Med J 53: 24-27.

11. Badia L, Lund VJ (2001) A case of rhinoscleroma treated with ciprofloxacin. The Journal of Laryngology \& Otology 115: 220-222.

12. Adekanye AG, Omotoso AJ, Emanghe UE, Umana AN (2015) Rhinoscleroma with malignancy- a case report \& review of literature. Trop Med Surg 3: 187. 